\title{
Failure to shorten the diagnostic delay in two ultra-orphan diseases (mucopolysaccharidosis types I and III): potential causes and implications
}

\author{
Gé-Ann Kuiper $^{\dagger}$, Olga L. M. Meijer ${ }^{\dagger}$, Eveline J. Langereis and Frits A. Wijburg ${ }^{*}$
}

\begin{abstract}
Background: Rare diseases are often un- or misdiagnosed for extended periods, resulting in a long diagnostic delay that may significantly add to the burden of the disease. An early diagnosis is particularly essential if a diseasemodifying treatment is available. The purpose of this study was to assess the extent of the diagnostic delay in the two ultra-rare diseases, i.e., mucopolysaccharidosis I (MPS I) and III (MPS III), both of which are lysosomal storage disorders with different phenotypic severities (MPS 1 is characterized by the severe Hurler and the more attenuated non-Hurler phenotypes, MPS III is characterized by the severe rapidly progressing (RP) phenotype and more attenuated slowly progressing (SP) phenotype). We investigated whether the diagnostic delay changed over the previous decades.
\end{abstract}

Results: The diagnostic delay, which is defined as the time between the first visit to a medical doctor for diseaserelated symptoms and the final diagnosis, was assessed using telephone interviews with patients diagnosed between 1988 and 2017 and/or their parents or legal guardian(s). In addition, the medical charts were reviewed. For MPS I $(n=$ 29), the median diagnostic delay was 8 months (range 1-24 months) for Hurler patients and 28 months (range 2147 months) for non-Hurler patients. For MPS III ( $n=46)$, the median diagnostic delay was 33 months (range 1365 months). No difference was observed between the RP and SP phenotypic groups. Comparing the diagnostic delay over time using 5-year time intervals, no reduction in the diagnostic delay was observed for MPS I or MPS III.

Conclusions: In the Netherlands, the time to diagnosis for patients with MPS I and MPS III has not changed between 1988 and 2017, and an extensive delay still exists between the first visit to a medical doctor for disease-related symptoms and the final diagnosis. The numerous campaigns launched to increase awareness, leading to earlier diagnosis of these rare disorders, particularly of MPS I, have failed to achieve their goal. Robust selected screening protocols embedded in national guidelines and newborn screening for disorders that meet the criteria for population screening may be the only effective approaches for reducing the diagnostic delay.

Keywords: Mucopolysaccharidosis type I, Mucopolysaccharidosis type III, Diagnostic delay, Awareness, Rare diseases

\footnotetext{
* Correspondence: f.a.wijburg@amc.uva.nl

${ }^{\dagger}$ Equal contributors

Department of Pediatric Metabolic Diseases, Emma Children's Hospital and

Amsterdam Lysosome Center "Sphinx", Academic Medical Center,

Meibergdreef 9, 1105, AZ, Amsterdam, The Netherlands
}

(c) The Author(s). 2018 Open Access This article is distributed under the terms of the Creative Commons Attribution 4.0 International License (http://creativecommons.org/licenses/by/4.0/), which permits unrestricted use, distribution, and reproduction in any medium, provided you give appropriate credit to the original author(s) and the source, provide a link to the Creative Commons license, and indicate if changes were made. The Creative Commons Public Domain Dedication waiver (http://creativecommons.org/publicdomain/zero/1.0/) applies to the data made available in this article, unless otherwise stated. 


\section{Background}

Rare diseases with a prevalence of less than 1 in 2000 citizens (as defined by the European Commission; EC) often carry a high physical and psychological burden and impact the quality of life of the patients, parents and caregivers. More than 6000 rare diseases have been identified, and $>50 \%$ are present during childhood (https://ec.europa.eu/ health/rare_diseases/policy, http://www.eurordis.org/sites/ default/files/publications/Fact_Sheet_RD.pdf). During previous decades, public and non-public organizations have launched numerous initiatives to increase the awareness of rare diseases, and in 1999, rare diseases first appeared on the agenda of the EC, resulting in a set of regulations and policies focusing on improving the recognition and visibility of rare diseases (https://ec.europa.eu/health/rare_diseases/ policy, http://ec.europa.eu/health/archive/ph_overview/previous_programme/rare_diseases/raredis_wpgm99_en.pdf).

Due to their nature and the non-specific symptoms at presentation and during the early phases of the disease, rare diseases are often un- or misdiagnosed for extended periods, leading to a long diagnostic delay [1-4]. Patients may visit many different healthcare professionals and undergo multiple unnecessary investigations before the correct diagnosis is finally achieved [1-4]. This diagnostic odyssey may significantly add to the burden of the disease [1, 2, 4]. An early diagnosis is particularly essential if a disease- modifying treatment is available because the patients' outcome often depends on the timely initiation of treatment [5-7]. Finally, because approximately $80 \%$ of rare diseases are inherited, an early diagnosis may allow genetic counseling and informed decision-making in family planning (https://www.eurordis.org/sites/default/files/ publications/Fact_Sheet_RD.pdf).

To prevent unnecessarily delayed diagnoses, numerous campaigns have been launched to increase awareness of rare diseases. Many campaigns, such as the 'rare diseases day' initiative, which has become a yearly event in many countries worldwide, are of a general nature, raising awareness of the existence of 'rare diseases'. Other initiatives focus on specific diseases and promoting an early diagnosis, thereby allowing the timely initiation of treatment [8-10] (http://www.rarediseaseday.org/events/world). These campaigns are organized by patient advocacy groups, health care providers and pharmaceutical companies.

However, to the best of our knowledge, no studies have specifically investigated whether these campaigns have reduced the diagnostic delay. We investigated the time to diagnosis of two very rare, invariable progressive and severe, inborn errors of metabolism: mucopolysaccharidosis type I (MPS I; estimated birth prevalence 1:100,000) for which treatment has been available for more than 15 years, and mucopolysaccharidosis type III (MPS III; estimated

Table 1 Symptoms frequently observed in MPS I and MPS III patients and information regarding the different phenotypes and enzymatic subtypes

\begin{tabular}{|c|c|c|c|c|c|c|}
\hline Disease & OMIM & Enzyme deficiency & Storage material & Main clinical features & Treatment & Prevalence \\
\hline \multicolumn{7}{|c|}{ Mucopolysaccharidosis type 1 (MPS I) } \\
\hline $\begin{array}{l}\text { MPS I- Hurler } \\
\text { (MPS I-H) }\end{array}$ & 607,014 & $\begin{array}{l}\text { a-L-iduronidase } \\
\text { (IDUA) }\end{array}$ & $\begin{array}{l}\text { Dermatan } \\
\text { sulfate (DS) and } \\
\text { heparan sulfate } \\
\text { (HS) }\end{array}$ & $\begin{array}{l}\text { Progressive neurocognitive decline, hernias, facial } \\
\text { dysmorphisms, corneal clouding, stiff joints, } \\
\text { dysostosis multiplex, cardiac problems and } \\
\text { hepatosplenomegaly. Death in childhood if } \\
\text { untreated. }\end{array}$ & $\mathrm{HSCT}$ & $\begin{array}{l}1.07 / 1.19 \\
\text { per } \\
100.000 \\
\text { newborns }\end{array}$ \\
\hline $\begin{array}{l}\text { MPS I - Hurler- } \\
\text { Scheie (MPS I-H/S) }\end{array}$ & 607,015 & & & $\begin{array}{l}\text { Phenotype intermediate between MPS I-H and MPS } \\
\text { I-S. Can present with or without neuronopathic } \\
\text { disease. }\end{array}$ & $\begin{array}{l}\text { HSCT or } \\
\text { ERT }\end{array}$ & \\
\hline $\begin{array}{l}\text { MPS I-Scheie } \\
\text { (MPS I-S) }\end{array}$ & 607,016 & & & $\begin{array}{l}\text { Corneal clouding, stiff joints, mild dysostosis } \\
\text { multiplex. Normal intelligence en life expectancy. }\end{array}$ & ERT & \\
\hline \multicolumn{7}{|c|}{ Mucopolysaccharidosis type 3 (MPS III) } \\
\hline MPS IIIA & 252,900 & $\begin{array}{l}\text { Heparan N-sulfatase } \\
\text { (SGSH) }\end{array}$ & $\begin{array}{l}\text { Heparan sulfate } \\
(\mathrm{HS})\end{array}$ & $\begin{array}{l}\text { Progressive neurocognitive decline, behavioral } \\
\text { problems, sleep disturbances, progressive loss of } \\
\text { motor functions. Death in second or third decade of } \\
\text { life. Broad spectrum of disease severity. }\end{array}$ & $\begin{array}{l}\text { Not } \\
\text { available }\end{array}$ & $\begin{array}{l}1.52 / 1.89 \\
\text { per } \\
100.000 \\
\text { newborns }\end{array}$ \\
\hline MPS $\| B$ & 252,920 & $\begin{array}{l}\text { N-acetyl-a- } \\
\text { glucosaminidase } \\
\text { (NAGLU) }\end{array}$ & & & & \\
\hline MPS IIIC & 252,930 & $\begin{array}{l}\text { Acetyl CoA:a- } \\
\text { glucosaminide } N^{-} \\
\text {acetyltransferase } \\
\text { (HGSNAT) }\end{array}$ & & & & \\
\hline MPS IIID & 252,940 & $\begin{array}{l}N \text { - } \\
\text { acetylglucosamine } \\
\text { 6-sulfatase (GNS) }\end{array}$ & & & & \\
\hline
\end{tabular}


birth prevalence 1:60,000) for which treatment is under study (Table 1). Both disorders belong to the group of lysosomal storage disorders. We assessed whether the diagnostic delay has decreased over recent decades.

\section{Methods}

\section{Patients}

This single center study was conducted at the Academic Medical Center (AMC) in Amsterdam and involved interviews with patients and/or parents or legal guardian(s) of patients with MPS I and MPS III with a confirmed diagnosis since 1988. Before 1988, reliable data were unavailable. The data were verified and/or supplemented with chart reviews or data inquiries from the general practitioner (GP) and the medical specialist(s) visited prior to diagnosis. Our center is a center of expertise for MPS I and MPS III in the Netherlands.

All MPS I and MPS III patients were included regardless of the phenotype. Table 1 presents the symptoms frequently observed in MPS I and MPS III patients and information regarding the different phenotypes and enzymatic subtypes [11-15]. The phenotypes were assessed by an experienced clinician (FAW) based on the available clinical data. Only patients with a diagnosis confirmed by enzymatic testing and/or a mutation analysis were included. Patients were only included if the diagnostic studies leading to the final diagnosis were based on the clinical symptoms. Patients who underwent diagnostic studies because of an affected family member were excluded. All patients and/or their parents or legal guardians provided informed consent for this study. The study proposal was reviewed by the Medical Ethics Committee of the AMC, who deemed that formal ethical approval was not necessary for this study.

\section{Data collection}

The data were collected using structured telephone interviews with patients and/or the patients' parents or legal guardian(s). The following variables were recorded:

- Year/month of first visit to the GP for a symptom that was, in hindsight, likely related to MPS I/MPS III.

- Year/month of first referral visit to a medical specialist for a symptom that was, in hindsight, likely related to MPS I/MPS III.

- Year/month of the confirmatory diagnosis, which was defined by the first demonstration of deficient enzyme activity or the presence of disease causing mutations.

From each of these visits, the following data were recorded:

- MPS I/MPS III-related symptom leading to the visit.

- Other MPS I/MPS III-related symptoms present at that time point.
Table 2 Disease-related symptoms for MPS I and MPS III

\begin{tabular}{ll}
\hline Disease-related symptoms & \\
\hline Mucopolysaccharidosis type I & Mucopolysaccharidosis \\
& type Ill \\
\hline Hernias & Developmental delay or \\
- Inguinal hernia & decline \\
- Umbilical hernia & - Neurocognitive \\
& functions \\
& - Motor functions \\
Ear, nose, throat problems & Behavioral problems \\
- Frequent upper airway infections & - Hyperactivity/ \\
- Obstructive sleep apneas or excessive & restlessness \\
snoring during sleep & - Aggression \\
- Tympanostomy tubes & - Anxiety \\
- Adenoidectomy & - Autistic behaviors \\
- Tonsillectomy & - Other \\
Gastro-intestinal problems & Dysmorphic features \\
- Hepatosplenomegaly & - Coarse facial features \\
Cardiac problems & - Coarse hair \\
- Cardiomyopathy & - Hirsutism \\
- Valvular dysfunction & - Other \\
Skeletal and joint problems & \\
- Joint stiffness & Ear, nose, throat problems \\
- Skeletal deformities & - Frequent upper airway \\
- Kyphosis & infections \\
- Hip dysplasia & - Frequent ear infections \\
- Bullet shaped metacarpals & - Hearing problems \\
- Stunted growth of the long bones & - Tympanostomy tubes \\
- Broad oar shaped ribs & - Adenoidectomy \\
- Short stature & - Tonsillectomy \\
- Carpal tunnel syndrome & Gastro-intestinal problems \\
- Trigger fingers & - Frequent diarrhea \\
- Tendon shortening & - Hepatomegaly \\
- Early arthrosis & - Other \\
Hydrocephalus & \\
Corneal clouding & Sleeping problems \\
Dysmorphic features & Seizures \\
- Frontal bossing & Hernias \\
- Depressed nasal bridge & - Inguinal hernia \\
- Full lips & - Umbilical hernia \\
- Macroglossia & \\
Developmental delay & \\
\hline & \\
&
\end{tabular}

- Type of medical specialist visited at first referral for a disease-related symptom.

- Type of medical specialist who made the diagnosis.

MPS I and MPS III disease-related symptoms are presented in Table 2.

\section{Statistical analyses}

The statistical analyses were performed using SPSS software for Windows (version 23.0, SPSS Inc., Chicago, Illinois, USA). Non-parametric Mann-Whitney $U$ tests were performed to assess the significant differences in the time between the first visit to the GP and diagnosis and the time between the first visit to a medical specialist and the final diagnosis within the cohort of MPS I patients and between the Hurler and non-Hurler patients. 
The same analyses were performed for the RP and SP MPS III patients.

To assess whether the diagnostic delay changed over time, the MPS I and MPS III patients were divided into different groups based on the year of diagnosis using a 5-year time interval. Non-parametric Kruskall-Wallis tests were performed to assess the significant differences among these groups.

\section{Results}

\section{MPS I and MPS III patient characteristics}

Thirty-two MPS I patients met the inclusion criteria; of these patients, three were excluded (two patients did not consent, and one was lost to follow-up). From the group of MPS III patients, 53 patients met the inclusion criteria, and 7 of these patients were lost to follow-up. The characteristics of the patients included in the study are provided in Table 3. At the time of this study, one male MPS IIIA patient (aged 4 years and 9 months) was considered too young to reliably predict the phenotypic severity.

\section{MPS I: First visit to the GP for an MPS I-related symptom}

Sixteen of the 29 MPS I patients first visited a GP for an MPS I-related symptom and were subsequently referred to a medical specialist. Eleven patients were directly seen by a medical specialist for MPS I-related symptoms without a prior visit to the GP, and this information was unclear for 2 patients. Due to the small group size, no further analyses of the first visit to the GP were performed.

Table 3 Characteristics of the MPS I and MPS III patients. At the time of this study, one of the MPS III patients (aged 4 years and 9 months) was considered too young to determine the phenotypic severity

\begin{tabular}{llll}
\hline Patient characteristics & & \\
\hline MPS I & N & MPS III & N \\
\hline Total number of patients & 29 & Total number of patients & 46 \\
Male & 15 & Male & 27 \\
Female & 14 & Female & 19 \\
MPS I phenotype & & MPS III subtype & 28 \\
Hurler & 20 & MPS IIIA & 9 \\
Non-Hurler & 9 & MPS IIIB & 9 \\
& & MPS IIIC & \\
& & MPS III phenotype & 16 \\
& & Rapidly progressing (RP) MPS III & 16 \\
& & Slowly progressing (SP) MPS III & 28 \\
& & Unknown & 1 \\
\hline
\end{tabular}

Table 4 Characteristics of the entire group of MPS I patients at the first visit to a medical specialist for an MPS I-related symptom as specified for the MPS I Hurler and non-Hurler patients

\begin{tabular}{|c|c|c|c|c|c|c|}
\hline \multicolumn{7}{|l|}{ First visit to a medical specialist } \\
\hline & \multicolumn{2}{|c|}{ All MPS I } & \multicolumn{2}{|c|}{ Hurler } & \multicolumn{2}{|c|}{ Non-Hurler } \\
\hline Number of patients & \multicolumn{2}{|l|}{29} & \multicolumn{2}{|l|}{20} & \multicolumn{2}{|l|}{9} \\
\hline \multicolumn{7}{|l|}{ Age at first visit (months) } \\
\hline Median & \multicolumn{2}{|l|}{4} & \multicolumn{2}{|l|}{3} & \multicolumn{2}{|l|}{12} \\
\hline Range & \multicolumn{2}{|c|}{$0-54$} & \multicolumn{2}{|c|}{$0-20$} & \multicolumn{2}{|c|}{$0-54$} \\
\hline Specialism of 1st referral & Nr. & $\%$ & Nr. & $\%$ & $\mathrm{Nr}$. & $\%$ \\
\hline Ear, nose, and throat specialist & 3 & $10 \%$ & 2 & $10 \%$ & 1 & $11 \%$ \\
\hline General pediatrician & 20 & $69 \%$ & 14 & $70 \%$ & 6 & $67 \%$ \\
\hline Orthopedic surgeon & 2 & $7 \%$ & 1 & $5 \%$ & 1 & $11 \%$ \\
\hline Pediatric surgeon & 3 & $10 \%$ & 2 & $10 \%$ & 1 & $11 \%$ \\
\hline Pediatric cardiologist & 1 & $3 \%$ & 1 & $5 \%$ & 0 & $0 \%$ \\
\hline Symptom leading to 1st referral & Nr. & $\%$ & Nr. & $\%$ & $\mathrm{Nr}$ & $\%$ \\
\hline Recurrent airway infections & 7 & $24 \%$ & 5 & $25 \%$ & 2 & $22 \%$ \\
\hline Upper airway obstruction & 3 & $10 \%$ & 2 & $10 \%$ & 1 & $11 \%$ \\
\hline Inguinal/umbilical hernia & 4 & $14 \%$ & 3 & $15 \%$ & 1 & $11 \%$ \\
\hline Hydrocephalus & 2 & $7 \%$ & 1 & $5 \%$ & 1 & $11 \%$ \\
\hline Hepatosplenomegaly & 1 & $3 \%$ & 1 & $5 \%$ & 0 & $0 \%$ \\
\hline Kyphosis/hip dysplasia & 3 & $10 \%$ & 2 & $10 \%$ & 1 & $11 \%$ \\
\hline Joint stiffness & 1 & $3 \%$ & 0 & $0 \%$ & 1 & $11 \%$ \\
\hline Facial features & 4 & $14 \%$ & 4 & $20 \%$ & 0 & $0 \%$ \\
\hline Hearing problems & 1 & $3 \%$ & 1 & $5 \%$ & 0 & $0 \%$ \\
\hline Growth delay & 2 & $7 \%$ & 1 & $5 \%$ & 1 & $11 \%$ \\
\hline Developmental delay & 1 & $3 \%$ & 0 & $0 \%$ & 1 & $11 \%$ \\
\hline $\begin{array}{l}\text { Other MPS I-related } \\
\text { symptoms at 1st referral }\end{array}$ & $\mathrm{Nr}$ & $\%$ & Nr. & $\%$ & $\mathrm{Nr}$ & $\%$ \\
\hline Recurrent airway infections & 7 & $24 \%$ & 4 & $20 \%$ & 3 & $33 \%$ \\
\hline Upper airway obstruction & 10 & $34 \%$ & 9 & $45 \%$ & 1 & $5 \%$ \\
\hline Inguinal/umbilical hernia & 8 & $28 \%$ & 6 & $30 \%$ & 2 & $10 \%$ \\
\hline Hydrocephalus & 2 & $7 \%$ & 2 & $10 \%$ & 0 & $0 \%$ \\
\hline Hepatosplenomegaly & 2 & $7 \%$ & 1 & $5 \%$ & 1 & $5 \%$ \\
\hline Joint stiffness & 3 & $10 \%$ & 2 & $10 \%$ & 1 & $11 \%$ \\
\hline Facial features & 4 & $14 \%$ & 2 & $10 \%$ & 2 & $10 \%$ \\
\hline Hearing problems & 6 & $21 \%$ & 4 & $20 \%$ & 2 & $10 \%$ \\
\hline Vision problems & 1 & $3 \%$ & 0 & $0 \%$ & 1 & $5 \%$ \\
\hline Developmental delay & 5 & $5 \%$ & 2 & $10 \%$ & 3 & $15 \%$ \\
\hline Growth delay & 1 & $3 \%$ & 0 & $0 \%$ & 1 & $5 \%$ \\
\hline
\end{tabular}

The sums of the percentages of each item may not equal $100 \%$ because the percentages represent rounded values

\section{MPS I: First visit to a medical specialist for an MPS I- related symptom}

The MPS I patients first visited a medical specialist for an MPS I-related symptom at a median age of 4 months (range 0 - 54 months; median age: MPS I Hurler patients 3 months (range $0-20$ months) and MPS I non- 
Hurler patients 12 months (range 0 - 54 months)) (Table 4).

Both the Hurler and non-Hurler patients were first seen by a general pediatrician (69\%), and recurrent airway infections were the most common reason for these visits. Additional MPS I-related symptoms that were present at the time of the first visit to a medical specialist are presented in Table 4.

\section{MPS I: Time to diagnosis}

The median age at diagnosis of all MPS I patients was 12 months (range 5 - 151 months) (Table 5). The Hurler patients were diagnosed at a significantly younger age (11 months (range 5 - 31 months) than the non-Hurler patients (57 months (range $5-151$ months) $(p=0.005)$ (Fig. 1a). The diagnosis of MPS I was most often made by a general pediatrician (45\%), followed by a pediatrician specialized in inborn errors of metabolism (IEM) (31\%).

The median delay between the first visit to a medical specialist and the final diagnosis for the entire group was 9 months (range 1 - 147; median delay: Hurler patients 8 months (range $1-24$ months) and non-Hurler patients 28 months (range $2-147$ months; the difference between the Hurler and non-Hurler patients was not significant) (Fig. 1b).

To assess whether the diagnostic delay changed over time, the patients were divided into different subgroups based on the year of the diagnosis using a 5-year time interval. Over the study period from 1988 to 2017, no significant reduction in the diagnostic delay was

Table 5 Characteristics of the entire group of MPS I patients, MPS I Hurler patients and non-Hurler patients at the time of diagnosis

Final diagnosis of MPS I

\begin{tabular}{|c|c|c|c|c|c|c|}
\hline & \multicolumn{2}{|c|}{$\begin{array}{l}\text { All MPS } \\
\text { I }\end{array}$} & \multicolumn{2}{|c|}{ Hurler } & \multicolumn{2}{|c|}{ Non-Hurler } \\
\hline Number of patients & \multicolumn{2}{|l|}{29} & \multicolumn{2}{|l|}{20} & \multicolumn{2}{|l|}{9} \\
\hline \multicolumn{7}{|l|}{ Age at diagnosis (months) } \\
\hline Median & \multicolumn{2}{|l|}{12} & \multicolumn{2}{|l|}{11} & \multicolumn{2}{|l|}{57} \\
\hline Range & \multicolumn{2}{|c|}{$5-151$} & \multicolumn{2}{|c|}{$5-31$} & \multicolumn{2}{|c|}{$5-151$} \\
\hline \multicolumn{7}{|l|}{$\begin{array}{l}\text { Delay medical specialist - diagnosis } \\
\text { (months) }\end{array}$} \\
\hline Median & \multicolumn{2}{|l|}{9} & \multicolumn{2}{|l|}{8} & \multicolumn{2}{|l|}{28} \\
\hline Range & \multicolumn{2}{|c|}{$1-147$} & \multicolumn{2}{|c|}{$1-24$} & \multicolumn{2}{|c|}{$2-147$} \\
\hline Diagnosing specialist & Nr. & $\%$ & Nr. & $\%$ & $\mathrm{Nr}$ & $\%$ \\
\hline General pediatrician & 13 & $45 \%$ & 10 & $50 \%$ & 3 & $33 \%$ \\
\hline Clinical geneticist & 4 & $14 \%$ & 3 & $15 \%$ & 1 & $11 \%$ \\
\hline Pediatrician specialized in IEM & 9 & $31 \%$ & 5 & $25 \%$ & 4 & $44 \%$ \\
\hline Ophthalmologist & 2 & $7 \%$ & 2 & $10 \%$ & 0 & $0 \%$ \\
\hline Rheumatologist & 1 & $3 \%$ & 0 & $0 \%$ & 1 & $11 \%$ \\
\hline
\end{tabular}

The sums of the percentages of each item may not equal $100 \%$ because the percentages represent rounded values. IEM: inborn errors of metabolism observed (Fig. 2a). In addition, no significant differences were observed in the time between the first visit to the medical specialist and the final diagnosis (Fig. 2b). When performing the same analyses only for the group of Hurler patients, no differences were observed in the median age at diagnosis and the median time between the first visit to the medical specialist and diagnosis over time (Fig. $2 \mathrm{~b}$ and d).

\section{MPS III: First visit to the GP for an MPS III-related symptom}

Almost all MPS III patients (45 of the 46) first visited a GP for an MPS III-related symptom (Table 6). The median age at the first visit for the entire group was 22 months (range $1-84$ months): 16 months for the $\mathrm{RP}$ patients (range $1-33$ months) and 24 months for the SP patients (range $1-84$ months). Upper airway infections and middle ear problems were the most frequent symptoms leading to the visit to the GP. Other symptoms leading to the visit to the GP and additional MPS III-related symptoms present at that time are presented in Table 6.

\section{MPS III: First visit to a medical specialist for an MPS III- related symptom}

The median age at the first visit to a medical specialist for an MPS III-related symptom was 28 months in the MPS III patients (range $2-171$ months; median age: 19 months in RP patients (range 6 39 months) and 30 months in SP patients (range 2 171 months)) (Table 7). The patients were most often referred to an ear, nose, and throat (ENT) specialist (65\%), and $61 \%$ of the cases subsequently underwent an adenotonsillectomy or placement of tympanostomy tubes (81\% of the RP patients and $52 \%$ of SP patients). In most patients, other MPS III-related symptoms were already present at the time of the first visit to the medical specialist, including developmental delay, behavioral and sleeping problems, dysmorphic features, hernias and recurrent episodes of unexplained diarrhea.

\section{MPS III: Time to diagnosis}

The final diagnosis was established at a median age of 62 months, with a range of 20 to 522 months (Table 8). As shown in Fig. 3a, the RP patients were significantly younger at the time of diagnosis (54 months, range $34-79$ months) than the SP patients $(71$ months, range $20-522)(p<0.05)$. The patients were most often diagnosed by a clinical geneticist, followed by a general pediatrician or a pediatrician specializing in IEM.

The median delay between the first visit to the GP for an MPS III-related symptom and the final diagnosis in 

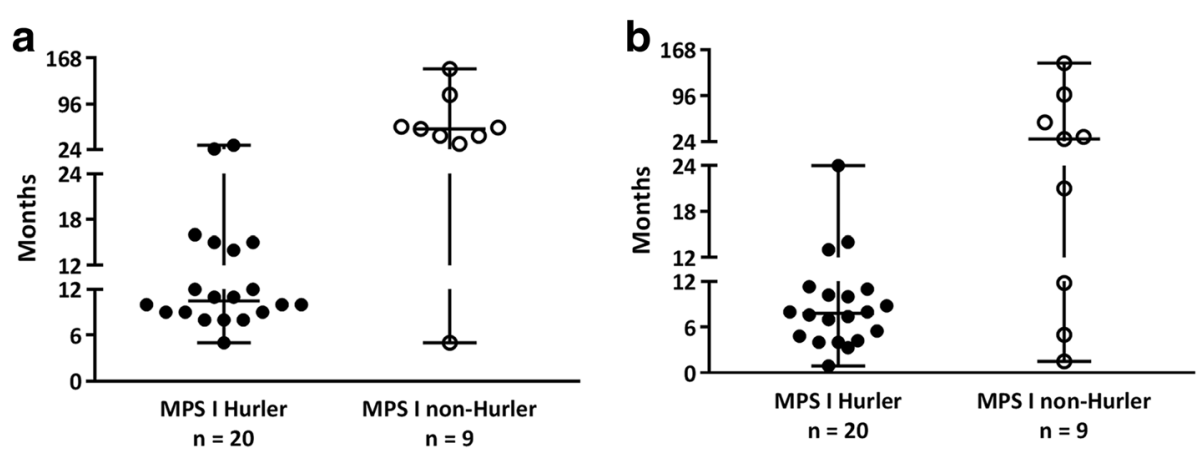

Fig. 1 a Age at diagnosis of the MPS I Hurler and non-Hurler patients. $\mathbf{b}$ Time between the first visit to a medical specialist for an MPS I-related symptom and final diagnosis in MPS I Hurler and non-Hurler patients. In all figures, time is presented in months

the entire group of MPS III patients was 39 months (range $2-438$ months), and no difference was observed among patient groups with varying disease severities (Fig. 3b). The median time between the first visit to a medical specialist for an MPS III-related symptom and the final diagnosis was 33 months (range 1 365 months). Similarly, no difference in delay was observed between the two phenotypic groups (Fig. 3c).

To assess whether the diagnostic delay changed over time, the MPS III patients were divided into different
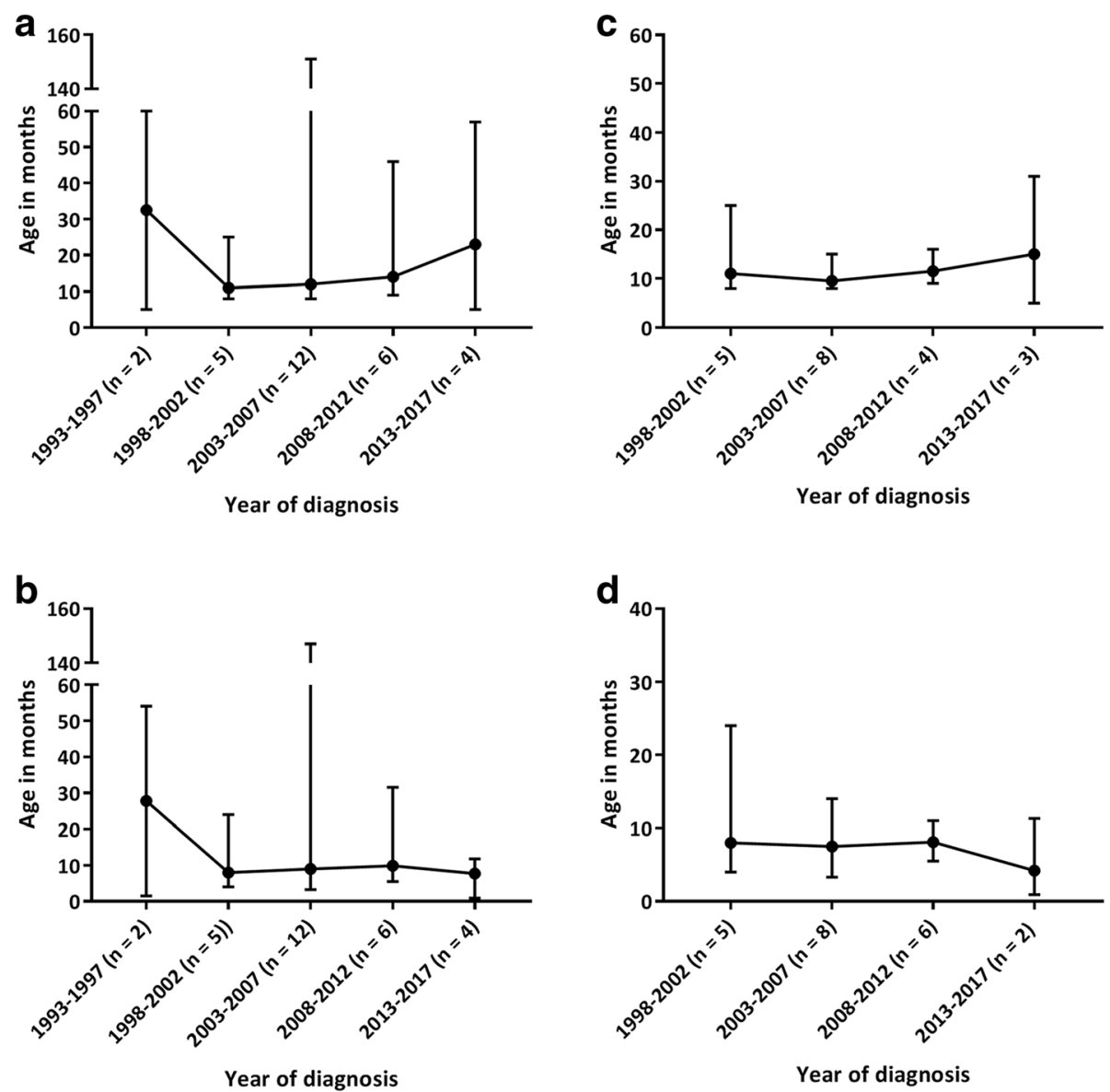

Fig. 2 a Age at diagnosis of the entire group of MPS I patients. b Time between the first visit to a medical specialist for an MPS I-related symptom and final diagnosis in the entire group of MPS I patients. c Age at diagnosis of the group of MPS I Hurler patients. $\mathbf{d}$ Time between the first visit to a medical specialist for an MPS I-related symptom and final diagnosis in the group of MPS I Hurler patients. In all figures, time is presented in months. Both MPS I and MPS I Hurler patients were divided into groups based on the year of diagnosis 
Table 6 Characteristics of the entire group of MPS III patients, RP MPS III patients and SP MPS III patients at the first visit to the GP for an MPS III-related symptom

\begin{tabular}{|c|c|c|c|c|c|c|}
\hline \multicolumn{7}{|l|}{ First visit to general practitioner } \\
\hline & \multicolumn{2}{|c|}{ All MPS III } & \multicolumn{2}{|c|}{ RP MPS III } & \multicolumn{2}{|c|}{ SP MPS III } \\
\hline Number of patients & \multicolumn{2}{|l|}{45} & \multicolumn{2}{|l|}{16} & \multicolumn{2}{|l|}{28} \\
\hline \multicolumn{7}{|l|}{ Age at 1st visit (months) } \\
\hline Median & \multicolumn{2}{|l|}{22} & \multicolumn{2}{|l|}{16} & \multicolumn{2}{|l|}{24} \\
\hline Range & \multicolumn{2}{|c|}{$1-84$} & \multicolumn{2}{|c|}{$1-33$} & \multicolumn{2}{|c|}{$1-84$} \\
\hline Symptom leading to 1st visit & Nr. & $\%$ & $\mathrm{Nr}$. & $\%$ & $\mathrm{Nr}$. & $\%$ \\
\hline Developmental delay & 9 & $20 \%$ & 2 & $13 \%$ & 6 & $21 \%$ \\
\hline Upper airway problems & 30 & $67 \%$ & 12 & $75 \%$ & 18 & $64 \%$ \\
\hline Diarrhea & 1 & $2 \%$ & 0 & $0 \%$ & 1 & $4 \%$ \\
\hline Liver problems & 1 & $2 \%$ & 0 & $0 \%$ & 1 & $4 \%$ \\
\hline Seizures & 1 & $2 \%$ & 0 & $0 \%$ & 1 & $4 \%$ \\
\hline Inguinal/umbilical hernia & 3 & $7 \%$ & 2 & $13 \%$ & 1 & $4 \%$ \\
\hline Other MPS III-related symptoms at 1st presentation & Nr. & $\%$ & $\mathrm{Nr}$ & $\%$ & $\mathrm{Nr}$. & $\%$ \\
\hline Developmental delay & 15 & $33 \%$ & 3 & $19 \%$ & 12 & $43 \%$ \\
\hline Behavioral problems & 29 & $64 \%$ & 10 & $63 \%$ & 18 & $64 \%$ \\
\hline Dysmorphic features & 27 & $60 \%$ & 11 & $69 \%$ & 15 & $54 \%$ \\
\hline Upper airway problems & 10 & $22 \%$ & 2 & $13 \%$ & 7 & $25 \%$ \\
\hline Diarrhea & 23 & $51 \%$ & 11 & $69 \%$ & 11 & $39 \%$ \\
\hline Sleeping disturbances & 21 & $47 \%$ & 9 & $56 \%$ & 11 & $39 \%$ \\
\hline Inguinal/umbilical hernia & 11 & $24 \%$ & 8 & $50 \%$ & 3 & $11 \%$ \\
\hline
\end{tabular}

One of the SP MPS III patients did not visit the GP before receiving a referral to a medical specialist. One of the patients was considered too young to determine the phenotypic severity at the time of this study. The sums of the percentages of each item may not equal $100 \%$ because the percentages represent rounded values

groups based on the year of the diagnosis using a 5-year time interval. Although a trend of diagnosing at a younger age was observed over time (Fig. 4a), no significant differences were observed between the cohorts of patients diagnosed in different time intervals. Similarly, the time between the first visit to the GP for an MPS III-related symptom and the time of the final diagnosis (Fig. $4 \mathrm{~b}$ ) and the time between the first visit to a medical specialist and the time of the final diagnosis (Fig. 4c) were not significantly reduced during the study period from 1988 to 2017 . Further analyses of the RP and SP patients did not reveal any differences over time (data not shown).

\section{Discussion}

This study is the first to report the diagnostic odyssey in MPS I and MPS III patients in the Netherlands. We demonstrate the presence of a substantial diagnostic delay in both MPS I and MPS III patients without a reduction in the time between the first consultation with a medical doctor (GP or medical specialist) for diseaserelated symptoms and the time of the final diagnosis over a 20-year period.

In the Dutch healthcare system, patients, including children, are typically first seen by a GP, who may refer the patient to a medical specialist. Thus, the time to diagnosis after the visit to the GP was longer than the time between the visit to a medical specialist and the diagnosis. Remarkably, the longest diagnostic delay was observed after the first visit to a medical specialist, particularly in the MPS III patients.

The MPS I patients were diagnosed at a significantly younger age than the MPS III patients, which is most likely due to the early manifestation of the somatic symptoms $[11,16]$, leading to earlier medical attention and referral. MPS I patients with the severe Hurler phenotype were diagnosed at a significantly younger age than the non-Hurler patients. The median age at diagnosis in the Hurler patients was comparable to that reported in previous studies [2, 17-19]. However, the more attenuated non-Hurler patients in our cohort were diagnosed at an earlier age than that reported in other studies [14, 17-20]. This finding may be due to the relatively small sample size of non-Hurler patients in our cohort. The lack of a decrease in the time to diagnosis over the previous two decades is disappointing and worrisome for two reasons. First, an early diagnosis allows for the early initiation of treatment and better disease outcomes. Treatment with hematopoietic cell transplantation (HCT) for MPS I Hurler was first shown to be effective in halting or 
Table 7 Characteristics of the entire group of MPS III patients, RP MPS III patients and SP MPS III patients at the first visit to a medical specialist for an MPS III-related symptom

\begin{tabular}{|c|c|c|c|c|c|c|}
\hline \multicolumn{7}{|l|}{ First visit to a medical specialist } \\
\hline & \multicolumn{2}{|c|}{$\begin{array}{l}\text { All MPS } \\
\text { III }\end{array}$} & \multicolumn{2}{|c|}{$\begin{array}{l}\text { RP MPS } \\
\text { III }\end{array}$} & \multicolumn{2}{|c|}{$\begin{array}{l}\text { SP MPS } \\
\|I\|\end{array}$} \\
\hline Number of patients & \multicolumn{2}{|l|}{46} & \multicolumn{2}{|l|}{16} & \multicolumn{2}{|l|}{29} \\
\hline \multicolumn{7}{|l|}{ Age at 1st visit (months) } \\
\hline Median & \multicolumn{2}{|l|}{28} & \multicolumn{2}{|l|}{19} & \multicolumn{2}{|l|}{30} \\
\hline Range & \multicolumn{2}{|c|}{$2-171$} & \multicolumn{2}{|c|}{$6-39$} & \multicolumn{2}{|c|}{$2-171$} \\
\hline Specialism of 1st referral & Nr. & $\%$ & & $\%$ & Nr. & $\%$ \\
\hline Ear, nose, and throat specialist & 30 & $65 \%$ & 13 & $81 \%$ & 16 & $55 \%$ \\
\hline General pediatrician & 7 & $15 \%$ & 1 & $6 \%$ & 6 & $21 \%$ \\
\hline Pediatric neurologist & 2 & $4 \%$ & 0 & $0 \%$ & 2 & $7 \%$ \\
\hline Pediatric surgeon & 4 & $9 \%$ & 2 & $13 \%$ & 2 & $7 \%$ \\
\hline Pediatric cardiologist & 1 & $2 \%$ & 0 & $0 \%$ & 1 & $3 \%$ \\
\hline Pediatric psychiatrist & 2 & $4 \%$ & 0 & $0 \%$ & 2 & $7 \%$ \\
\hline Symptom leading to 1st referral & $\mathrm{Nr}$. & $\%$ & Nr. & $\%$ & Nr. & $\%$ \\
\hline Developmental delay & 7 & $15 \%$ & 0 & $0 \%$ & 7 & $24 \%$ \\
\hline Upper airway problems & 2 & $4 \%$ & 1 & $6 \%$ & 1 & $3 \%$ \\
\hline $\begin{array}{l}\text { Adenotonsillectomy/tympanostomy } \\
\text { tubes }\end{array}$ & 29 & $63 \%$ & 13 & $81 \%$ & 15 & $52 \%$ \\
\hline Diarrhea & 1 & $2 \%$ & 0 & $0 \%$ & 1 & $3 \%$ \\
\hline Liver problems & 1 & $2 \%$ & 0 & $0 \%$ & 1 & $3 \%$ \\
\hline Seizures & 1 & $2 \%$ & 0 & $0 \%$ & 1 & $3 \%$ \\
\hline Correction Inguinal/umbilical hernia & 4 & $9 \%$ & 2 & $13 \%$ & 2 & $7 \%$ \\
\hline Cardiac murmur & 1 & $2 \%$ & 0 & $0 \%$ & 1 & $3 \%$ \\
\hline $\begin{array}{l}\text { Other MPS III-related symptoms at 1st } \\
\text { referral }\end{array}$ & $\mathrm{Nr}$. & $\%$ & Nr. & $\%$ & Nr. & $\%$ \\
\hline Developmental delay & 21 & $46 \%$ & 10 & $63 \%$ & 10 & $34 \%$ \\
\hline Behavioral problems & 35 & $76 \%$ & 14 & $88 \%$ & 20 & $69 \%$ \\
\hline Dysmorphic features & 29 & $63 \%$ & 11 & $69 \%$ & 17 & $59 \%$ \\
\hline Upper airway problems & 10 & $22 \%$ & 1 & $6 \%$ & 9 & $31 \%$ \\
\hline Diarrhea & 23 & $50 \%$ & 11 & $69 \%$ & 11 & $38 \%$ \\
\hline Sleeping disturbances & 21 & $46 \%$ & 9 & $56 \%$ & 12 & $41 \%$ \\
\hline Inguinal/umbilical hernia & 11 & $24 \%$ & 9 & $56 \%$ & 2 & $7 \%$ \\
\hline
\end{tabular}

The sums of the percentages of each item may not equal $100 \%$ because the percentages represent rounded values

preventing the cognitive decline in the early 1980s and is currently the treatment of choice for this group of patients. Earlier HCT leads to better outcomes [5, 21, 22]. In addition, intravenous enzyme replacement therapy (ERT) is the treatment of choice for MPS I patients with a non-Hurler phenotype, and studies have demonstrated that an early start of treatment is beneficial $[6,7,23,24]$. Second, to reduce the diagnostic delay and promote early diagnosis, numerous MPS I awareness campaigns have been launched, particularly after the introduction of ERT for the treatment of the
Table 8 Characteristics of the entire group of MPS III patients, RP MPS III patients and SP MPS III patients at the time of diagnosis

\begin{tabular}{|c|c|c|c|c|c|c|}
\hline \multicolumn{7}{|l|}{ Final diagnosis of MPS III } \\
\hline & \multicolumn{2}{|c|}{ All MPS III } & \multicolumn{2}{|c|}{ RP MPS III } & \multicolumn{2}{|c|}{ SP MPS III } \\
\hline Number of patients & \multicolumn{2}{|l|}{46} & \multicolumn{2}{|l|}{16} & \multicolumn{2}{|l|}{29} \\
\hline \multicolumn{7}{|l|}{ Age at diagnosis (months) } \\
\hline Median & \multicolumn{2}{|l|}{62} & \multicolumn{2}{|l|}{54} & \multicolumn{2}{|l|}{71} \\
\hline Range & \multicolumn{2}{|c|}{$20-522$} & \multicolumn{2}{|c|}{$34-79$} & \multicolumn{2}{|c|}{$20-522$} \\
\hline \multicolumn{7}{|c|}{ Delay general practitioner - diagnosis (months) } \\
\hline Median & \multicolumn{2}{|l|}{39} & \multicolumn{2}{|l|}{39} & \multicolumn{2}{|l|}{42} \\
\hline Range & \multicolumn{2}{|c|}{$2-438$} & \multicolumn{2}{|c|}{$6-76$} & \multicolumn{2}{|c|}{$3-438$} \\
\hline \multicolumn{7}{|c|}{ Delay medical specialist - diagnosis (months) } \\
\hline Median & \multicolumn{2}{|l|}{33} & \multicolumn{2}{|l|}{33} & \multicolumn{2}{|l|}{41} \\
\hline Range & \multicolumn{2}{|c|}{$1-365$} & \multicolumn{2}{|c|}{$2-66$} & \multicolumn{2}{|c|}{$5-365$} \\
\hline Diagnosing specialist & Nr. & $\%$ & Nr. & $\%$ & Nr. & $\%$ \\
\hline Clinical geneticist & 16 & $35 \%$ & 5 & $31 \%$ & 11 & $38 \%$ \\
\hline General pediatrician & 13 & $28 \%$ & 5 & $31 \%$ & 8 & $28 \%$ \\
\hline Pediatrician specialized in IEM & 12 & $26 \%$ & 5 & $31 \%$ & 6 & $21 \%$ \\
\hline Pediatric neurologist & 4 & $9 \%$ & 1 & $6 \%$ & 3 & $10 \%$ \\
\hline Specialist for the mentally disabled & 1 & $2 \%$ & 0 & $0 \%$ & 1 & $3 \%$ \\
\hline
\end{tabular}

The sums of the percentages of each item may not equal 100\% because the percentages represent rounded values. IEM: inborn errors of metabolism

somatic symptoms in 2003. These campaigns included direct mailings to health care professionals in the Netherlands presenting the typical features of MPS I patients, expert lectures on early symptoms of MPS I at scientific meetings of relevant medical specialists (including pediatricians, ENT specialists, pediatric rheumatologists and pediatric neurologists) and exhibit booths of a pharmaceutical company commercially marketing ERT for MPS I (Genzyme Sanofi) providing educational material on lysosomal storage disorders, including MPS I, at major relevant medical conferences in the Netherlands. Our data indicate that these efforts have not led to a significant reduction in the time to an MPS I diagnosis.

In our cohort of MPS III patients, the diagnosis was established at a significantly younger age in the severe RP patients (age 54 months; 4 years and 6 months) than in the SP patients (age 71 months; 5 years and 11 months). However, the diagnostic process preceding the diagnosis did not differ between the two groups, and the age at final diagnosis is comparable to observations reported in other studies [25-27]. Although no disease-modifying treatment is currently available, several clinical trials, including intrathecal ERT and gene therapy, have recently been initiated for MPS III types A and B [28, 29]. An early diagnosis and early start of treatment before the onset of progressive cognitive deterioration are considered 

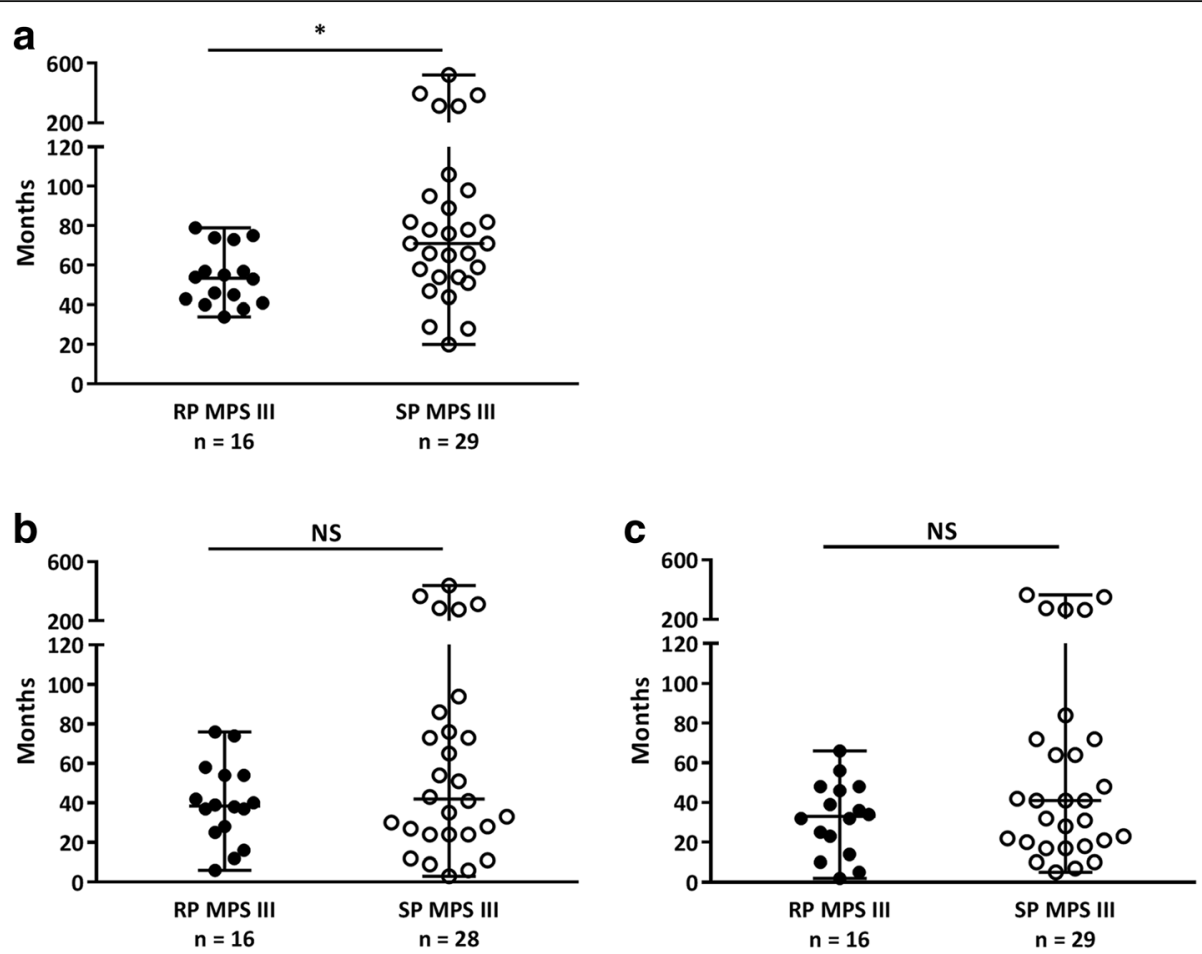

Fig. 3. a Age at diagnosis in the RP and SP MPS III patients. b Time between the first visit to the GP for an MPS III-related symptom and the final diagnosis (in months) in the RP and SP MPS III patients. c Time between the first visit to a medical specialist for an MPS III-related symptom and the final diagnosis (in months) in the RP and SP MPS III patients. ${ }^{*} p<0.05$; NS = non-significant

essential. Given that patients with the RP phenotype plateau in development by 30 months and exhibit rapid cognitive decline at $40-50$ months, a diagnosis should be made before the age of 3 years to allow the initiation of therapy at the optimal timing [13]. This goal, however, was only achieved in $9 \%$ of the patients in this study, and no decrease in age at diagnosis was observed over the previous 20 years.

Our study has some limitations. First, we defined diagnostic delay as the time between the first visit to a GP or medical specialist for a potential disease-related symptom and the final diagnosis, whereas diagnostic delay generally refers to the time between the onset of symptoms and diagnosis in other studies [14, 30, 31]. However, we consider the use of the time of symptom onset susceptible to a significant recall bias, whereas the time of the first visit to a medical doctor can be verified, thereby providing more reliable data. Second, our study has a retrospective design. Nevertheless, the amount of missing data was small, and the data could be verified in the medical records. In addition, due to the rarity of both disorders, a prospective design is not feasible. Third, the number of patients included in our study was small. Given that we were able to recruit almost all patients from the Netherlands diagnosed with MPS I and MPS III between 1988 and 2017, we assume that our data reliably represent the situation in our country.
Larger scale, multi-national, studies on the diagnostic delay in patients with MPS or other rare or ultra-rare diseases are needed to corroborate our findings. In Europe, such studies may be initiated by the recently established European Reference Networks for rare diseases (ERNs) (https://ec.europa.eu/health/ern_en). Finally, MPS I and MPS III are ultra-rare (ultra-orphan) diseases because they affect less than one person per 50,000 people (http://eur-lex.europa.eu/legal-content/EN/TXT/ ?uri=CELEX:32014R0536). The results of our study might not be applicable to relatively more common rare diseases affecting one person per $2000-50,000$ people.

The lack of a reduction in the diagnostic delay over time was previously reported for MPS I by d'Aco et al, based on data from an observational international MPS I registry [18]. In addition, a study investigating the time of diagnosis in Pompe disease, which is a lysosomal storage disease in which the timing of the start of therapy (ERT) is essential, to the surprise of the authors, also failed to demonstrate a reduction in the diagnostic delay despite improved diagnostic laboratory techniques allowing for a rapid diagnosis [32]. Multiple efforts to increase awareness of Pompe disease and expedite its diagnosis have been exerted globally over recent decades.

Determining why awareness campaigns for rare diseases fail to reduce the diagnostic delay in MPS I and III and Pompe disease is challenging. Due to the very low birth 


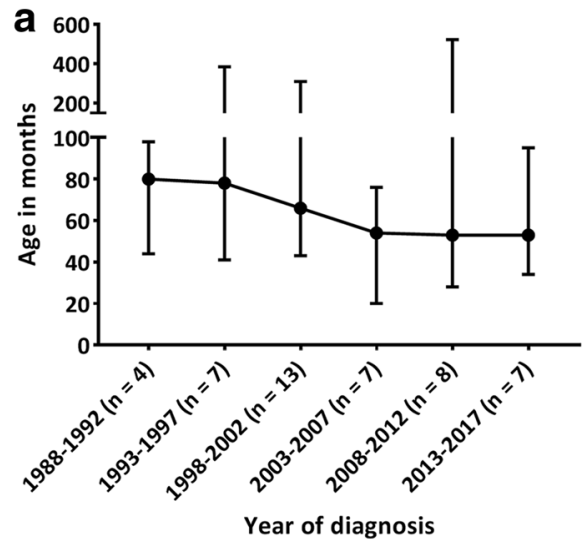

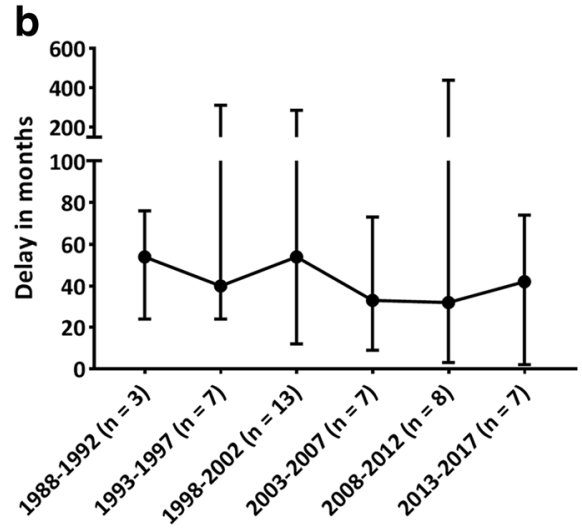

Year of diagnosis

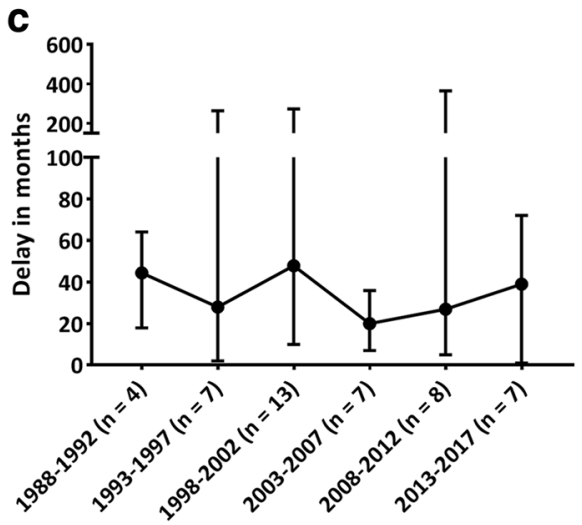

Year of diagnosis

Fig. 4 a Age at diagnosis in the MPS III patients. $\mathbf{b}$ Time between the first visit to a GP for an MPS II-related symptom and the final diagnosis. $\mathbf{c}$ Time between the first visit to a medical specialist for an MPS III-related symptom and the final diagnosis. In all figures, time is presented in months. The MPS III patients were divided into groups based on the year of diagnosis. One patient never visited the GP for an MPS III-related symptom

prevalence of these disorders, many specialists, including GPs, general pediatricians, orthopedic surgeons and ENT specialists, may visit with no or only one undiagnosed patient during their entire career. Awareness of specific (combinations of) symptoms of a (ultra) rare disease may be lacking when confronted with a patient (many) years after exposure to an awareness campaign. Long-lasting knowledge regarding the symptoms of (ultra) rare diseases can likely only be achieved by intensive repetitive learning, which is not a feasible option for all medical specialists. Furthermore, because most symptoms at presentation are not specific, considerable time is generally spent excluding more common disorders.

Several alternative strategies are possible. One strategy involves the selective screening of groups of patients with certain symptoms but without a diagnosis of the rare disease of interest. Such studies have been performed for MPS I and included studies investigating MPS screening in patients with previous surgical repair or the presence of inguinal and/or umbilical hernia in combination with pediatric ENT surgery and children visiting rheumatology, hand or skeletal dysplasia clinics (clinicaltrials.gov identifiers: NCT02095015, NCT01675674). Both trials have been terminated. To the best of our knowledge, these results have not been published, suggesting a failure to identify significant numbers of otherwise unrecognized patients. A study investigating screening patients under the age of 18 years with carpal tunnel syndrome for MPS also failed to detect patients with MPS [33]. The extremely low yield of screening certain groups of patients for an ultrarare disorder likely discourages participation, leading to the discontinuation of these programs. The yields of selective screening may improve when groups of patients are screened for a multitude of disorders, thus obviating the need of knowledge regarding specific rare disorders. Because the diagnostic approach in children with impaired cognitive development may significantly differ among health care systems in different regions of the world and obtaining an early diagnosis in patients with MPS III is very difficult, screening of children with an intellectual developmental disorder for several rare diseases may significantly reduce the diagnostic delay. A diagnostic algorithm for the identification of treatable causes of cognitive impairment has been proposed [34], and several publications 
have demonstrated the importance of an early metabolic screening in all patients with unexplained developmental delay $[35,36]$. In addition, a review by Cleary and Green [37] provided a guideline for the metabolic screening of patients with a developmental delay. The authors emphasize that IEMs can present with isolated developmental delay and that any regression of skills is suggestive of an IEM and warrants an intensive metabolic investigation. The slowing of cognitive development with a speech delay is one of the first symptoms of MPS III and often occurs before the age of 2.5 years; these symptoms could lead to an early diagnosis if these guidelines are followed. However, as the median age at diagnosis of patients with the most common RP phenotype is 54 months (range $34-79$ months) in our study, it is clear that these guidelines are not used in the Netherlands. Indeed, the current guideline by the Dutch Society for Pediatrics (NvK, 2005) recommends screening for IEMs only if additional symptoms are present and not in in the presence of isolated cognitive delay (https://www.nvk.nl/Portals/0/richtlijnen/mentale\%20retardatie/mentaleretardatie.pdf). Fortunately, a new guideline is currently under development.

An interesting option for the (near) future is computerassisted diagnosis, which can expedite the diagnosis of rare diseases. Artificial intelligence, deep learning and even a 3D facial analysis may assist clinicians during the diagnostic process, suggesting both diagnoses and appropriate investigations based on information in the electronic patient records [38-40].

Finally, newborn population screening (NBS) may ensure very early diagnosis in patients with rare diseases and should be considered if a disease meets at least the following criteria (first proposed by Wilson and Jungner in 1968) [41]: (a) the condition is an important health problem; (b) a suitable test for diagnosis is available; (c) a latent or early symptomatic state is recognizable; (d) the understanding of the condition's natural history is adequate; and (e) an acceptable treatment for patients with a recognized disease is available. Because MPS I is considered to meet these criteria, this disorder has been introduced in NBS programs in the USA and Taiwan [42] and will be introduced in the NBS panel in the Netherlands (https://zoek.officielebekendmakingen.nl/blg-775624.pdf). However, this will lead to new challenges, including the detection of pseudo deficiencies for MPS I, as well the challenges often associated with newborn screening such as uncertain diagnoses and the inability to predict the phenotype, which may lead to significant emotional burden [43-45]. MPS III is currently not considered eligible for NBS because no disease-modifying therapy is yet available.

\section{Conclusions}

In conclusion, we demonstrate that the time to diagnosis in patients with MPS I and MPS III has not changed between 1988 and 2017 in the Netherlands and a long delay between the first visit to a medical doctor for symptoms related to the disease and the final diagnosis is common. Therefore, campaigns to increase the awareness of rare diseases in general, and of MPS I specifically, failed to achieve this goal. This finding is likely due to the non-specific initial symptoms and the ultra-rare nature of both disorders. Because most medical doctors will probably visit with patients with these disorders never or only once during their entire career, it is questionable whether education of combinations of symptoms of specific (ultra) rare diseases will ever be effective. Robust selected screening protocols embedded in national guidelines may be the best alternative. Such guidelines may include urinary screening for glycosaminoglycans in all children with kyphosis and extensive screening for IEMs in all children with developmental delay, thus obviating the need for detailed knowledge regarding specific (ultra) rare diseases. Finally, NBS should be considered for those disorders that meet the criteria for population screening because this may be the only approach to guarantee a timely initiation of therapy in all patients with specific rare diseases.

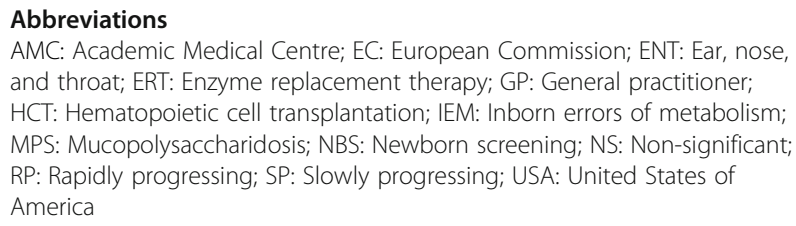

\section{Acknowledgments}

We thank all families for their willingness to contribute to this study and participate in our telephone survey.

\section{Funding}

This study was partially funded by a grant from Sanofi Genzyme, and by grants from the Foundation Kinderen en Kansen, Zabawas and the Rare Diseases Foundation. The funders had no role in the design and conduct of the study; collection, management, analysis, and interpretation of the data; preparation, review, or approval of the manuscript; and decision to submit the manuscript for publication.

\section{Availability of data and materials \\ The datasets used and/or analyzed during the current study are available from the corresponding author upon reasonable request.}

\section{Authors' contributions}

GK and OM designed the study; conducted the interviews with the patients, parents and/or caregivers; collected and analyzed the data; and wrote the manuscript. EL designed and supervised the study. FA designed and supervised the study and revised the manuscript. All authors read and approved the final manuscript.

\section{Ethics approval and consent to participate}

The study proposal was reviewed by the Medical Ethics Committee of the Academic Medical Center, who deemed that formal ethical approval was not necessary for this study. The patients and/or the patients' parents or legal guardians provided informed consent for this study.

Consent for publication

Not applicable. 


\section{Competing interests}

F.A. Wijburg has received honoraria for presentations and board meetings, travel expenses to meetings, and honoraria for consultancy work from Sanofi Genzyme and Shire HGT, and has received unrestricted educational grants and research grants from Sanofi Genzyme. G. Kuiper, O.L.M. Meijer and E.J. Langereis declare that they have no competing interests.

\section{Publisher's Note}

Springer Nature remains neutral with regard to jurisdictional claims in published maps and institutional affiliations.

\section{Received: 29 September 2017 Accepted: 4 December 2017}

\section{Published online: 08 January 2018}

\section{References}

1 Lisi EC, Gillespie S, Laney D, Ali N. Patients' perspectives on newborn screening for later-onset lysosomal storage diseases. Mol Genet Metab. 2016;119:109-14

2 de Ru MH, Bouwman MG, Wijburg FA, van Zwieten MCB. Experiences of parents and patients with the timing of Mucopolysaccharidosis type I (MPS I) diagnoses and its relevance to the ethical debate on newborn screening. Mol Genet Metab. 2012:107:501-7.

3 Bruni S, Lavery C, Broomfield A. The diagnostic journey of patients with mucopolysaccharidosis I: a real-world survey of patient and physician experiences. Mol Genet Metab Reports. 2016;8:67-73.

4 Zurynski Y, Deverell M, Dalkeith T, Johnson S, Christodoulou J, Leonard H, et al. Australian children living with rare diseases: experiences of diagnosis and perceived consequences of diagnostic delays. Orphanet J Rare Dis. 2017;12: 68

5 Aldenhoven M, Wynn RF, Orchard PJ, O'Meara A, Veys P, Fischer A, et al. Long-term outcome of hurler syndrome patients after hematopoietic cell transplantation: an international multicenter study. Blood. 2015;125:2164-72.

6 Gabrielli O, Clarke LA, Bruni S, Coppa GV. Enzyme-replacement therapy in a 5-month-old boy with attenuated presymptomatic MPS I: 5-year follow-up. Pediatrics. 2010;125:e183-7.

7 Laraway S, Breen C, Mercer J, Jones S, Wraith JE. Does early use of enzyme replacement therapy alter the natural history of mucopolysaccharidosis I? Experience in three siblings. Mol Genet Metab. 2013;109:315-6.

8 Zurynski Y, Frith K, Leonard H, Elliott E. Rare childhood diseases: how should we respond? Arch Dis Child. 2008;93:1071-4.

9 Hampton T. Rare disease research gets boost. JAMA. 2006;295:2836-8.

10 Schieppati A, Henter JI, Daina E, Aperia A. Why rare diseases are an important medical and social issue. Lancet. 2008;371:2039-41.

11 Neufeld EF, Muenzer J. The Mucopolysaccharidoses. In: Valle D, Beaudet A Vogelstein B, et al., editors. Metab Mol Bases Inherit Dis. New York: McGrawHill; 2014

12 Valstar MJ, Ruijter GJG, van Diggelen OP, Poorthuis BJ, Wijburg FA Sanfilippo syndrome: a mini-review. J Inherit Metab Dis. 2008:31:240-52.

13 Shapiro EG, Nestrasil I, Delaney KA, Rudser K, Kovac V, Nair N, et al. A prospective natural history study of Mucopolysaccharidosis type IIIA. J Pediatr. 2016;170:278-287e4

14 Vijay S, Wraith JE. Clinical presentation and follow-up of patients with the attenuated phenotype of mucopolysaccharidosis type I. Acta Paediatr. 2005; 94:872-7.

15 Cleary M, Wraith JE. The presenting features of mucopolysaccharidosis type IH (hurler syndrome). Acta Paediatr. 1995;84:337-9.

16 Muenzer J. Overview of the mucopolysaccharidoses. Rheumatology. 2011 50(Suppl 5):v4-12.

17 Pastores GM, Arn P, Beck M, Clarke JTR, Guffon N, Kaplan P, et al. The MPS I registry: design, methodology, and early findings of a global disease registry for monitoring patients with Mucopolysaccharidosis type I. Mol Genet Metab. 2007;91:37-47

18 D'Aco K, Underhill L, Rangachari L, Arn P, Cox GF, Giugliani R, et al. Diagnosis and treatment trends in mucopolysaccharidosis I: findings from the MPS I registry. Eur J Pediatr. 2012;171:911-9.

19 Beck M, Arn P, Giugliani R, Muenzer J, Okuyama T, Taylor J, et al. The natura history of MPS I: global perspectives from the MPS I registry. Genet Med American College of Medical Genetics and Genomics. 2014;16:759-65.

20 Thomas JA, Beck M, Clarke JTR, Cox GF. Childhood onset of Scheie syndrome, the attenuated form of mucopolysaccharidosis i. J Inherit Metab Dis. 2010;33:421-7.
21 Muenzer J, Wraith JE, Clarke LA. Mucopolysaccharidosis I: management and treatment guidelines. Pediatrics. 2009;123:19-29.

22 de Ru MH, Boelens JJ, Das AM, Jones SA, van der Lee JH, Mahlaoui N, et al. Enzyme replacement therapy and/or hematopoietic stem cell transplantation at diagnosis in patients with mucopolysaccharidosis type I: results of a European consensus procedure. Orphanet J Rare Dis. 2011;6:55.

23 Al-Sannaa NA, Bay L, Barbouth DS, Benhayoun Y, Goizet C, Guelbert N, et al. Early treatment with laronidase improves clinical outcomes in patients with attenuated MPS I: a retrospective case series analysis of nine sibships. Orphanet J Rare Dis Orphanet Journal of Rare Diseases. 2015;10:131.

24 Gabrielli O, Clarke LA, Ficcadenti A, Santoro L, Zampini L, Volpi N, et al. 12 year follow up of enzyme-replacement therapy in two siblings with attenuated mucopolysaccharidosis I: the important role of early treatment. BMC Med Genet BMC Medical Genetics. 2016;17:19.

25 Meyer A, Kossow K, Gal A, Mühlhausen C, Ullrich K, Braulke T, et al. Scoring evaluation of the natural course of mucopolysaccharidosis type IIIA (Sanfilippo syndrome type a). Pediatrics. American Academy of Pediatrics. 2007;120:e1255-61.

26 Héron B, Mikaeloff Y, Froissart R, Caridade G, Maire I, Caillaud C, et al. Incidence and natural history of mucopolysaccharidosis type III in France and comparison with United Kingdom and Greece. Am J Med Genet A. 2011;155A:58-68.

27 Delgadillo V, O'Callaghan M del M, Gort L, Coll MJ, Pineda M. Natural history of Sanfilippo syndrome in Spain. Orphanet J Rare Dis. 2013;8:189.

28 Hollak CEM, Wijburg FA. Treatment of lysosomal storage disorders: successes and challenges. J Inherit Metab Dis. 2014;37:587-98.

29 Parenti G, Andria G, Ballabio A. Lysosomal storage diseases: from Pathophysiology to therapy. Annu Rev Med. 2015;66:471-86.

30 Valstar MJ, Bruggenwirth HT, Olmer R, Wevers RA, Verheijen FW, Poorthuis BJ, et al. Mucopolysaccharidosis type IIIB may predominantly present with an attenuated clinical phenotype. J Inherit Metab Dis. 2010;33:759-67.

31 Valstar MJ, Neijs S, Bruggenwirth HT, Olmer R, Ruijter GJG, Wevers RA, et al. Mucopolysaccharidosis type IIIA: clinical spectrum and genotype-phenotype correlations. Ann Neurol. 2010;68:876-87.

32 Kishnani PS, Amartino HM, Lindberg C, Miller TM, Wilson A, Keutzer J. Timing of diagnosis of patients with pompe disease: data from the pompe registry. Am J Med Genet Part A. 2013;161:2431-43.

33 Nørmark MB, LA Kjaer N. Prevalence of Mucopolysaccharidosis types I, II, and $\mathrm{VI}$ in the pediatric and adult population with carpal tunnel syndrome (CTS). Retrospective and prospective analysis of patients treated for CTS. JIMD Rep. 2017; Epub ahead

34 Van Karnebeek CDM, Shevell M, Zschocke J, Moeschler JB, Stockler S. The metabolic evaluation of the child with an intellectual developmental disorder: diagnostic algorithm for identification of treatable causes and new digital resource. Mol Genet Metab. 2014;111:428-38.

35 Engbers HM, Berger R, Van Hasselt P, De Koning T, De Sain-Van Der Velden MGM, Kroes HY, et al. Yield of additional metabolic studies in neurodevelopmental disorders. Ann Neurol. 2008;64:212-7.

36 Sayson B, Popurs MAM, Lafek M, Berkow R, Stockler-Ipsiroglu S, van Karnebeek CDM. Retrospective analysis supports algorithm as efficient diagnostic approach to treatable intellectual developmental disabilities. Mol Genet Metab. 2015;115:1-9.

37 Cleary MA, Green A. Developmental delay: when to suspect and how to investigate for an inborn error of metabolism. Arch Dis Child. 2005;90:1128-32.

38 Cahan A, Cimino JJ. A learning health care system using computer-aided diagnosis. J Med Internet Res. 2017;19:1-12.

39 Chen Y, Argentinis E, Weber G. IBM Watson: how cognitive computing can be applied to big data challenges in life sciences research. Clin Ther. 2016; 38:688-701.

40 Gripp KW, Baker L, Telegrafi A, Monaghan KG. The role of objective facia analysis using FDNA in making diagnoses following whole exome analysis. Report of two patients with mutations in the BAF complex genes. Am J Med Genet Part A. 2016;170:1754-62.

41 Wilson JMG, Jungner G. Principles and practice of screening for disease. World Heal Organ. 1968;163

42 Schielen P, Kemper E, Gelb M. Newborn screening for Lysosomal storage diseases: a concise review of the literature on screening methods. Therapeutic Possibilities and Regional Programs Int J Neonatal Screen. 2017;3:6.

43 Wang RY, Bodamer OA, Watson MS, Wilcox WR. Lysosomal storage diseases: diagnostic confirmation and management of presymptomatic individuals. Genet Med The American College of Medical Genetics. 2011;13:457-84. 
44 Parini R, Deodato F, Di Rocco M, Lanino E, Locatelli F, Messina C, et al. Open issues in Mucopolysaccharidosis type I-hurler. Orphanet J Rare Dis. Orphanet Journal of Rare Diseases. 2017;12:112.

45 Burton BK, Charrow J, Hoganson GE, Waggoner D, Tinkle B, Braddock SR et al. Newborn screening for Lysosomal storage disorders in Illinois: the initial 15-month experience. J Pediatr. 2017;2017:1-6.

Submit your next manuscript to BioMed Central and we will help you at every step:

- We accept pre-submission inquiries

- Our selector tool helps you to find the most relevant journal

- We provide round the clock customer support

- Convenient online submission

- Thorough peer review

- Inclusion in PubMed and all major indexing services

- Maximum visibility for your research

Submit your manuscript at www.biomedcentral.com/submit 